\title{
Estado Docente, subsidiariedad y libertad de enseñanza. El proceso de privatización educacional en Chile desde la perspectiva de los actores (1973-1990)
}

\section{Estado Ensino, subsidiariedade e da liberdade de educação. Processo de formação privatização no Chile do ponto de vista dos agentes (1973-1990)}

Teaching State, subsidiarity and freedom of education. The process of educational privatization in Chile from the perspective of the actors (1973-1990)

Camila Pérez-Navarro ${ }^{1}$

Andrés Rojas-Murphy²

DOI: http://dx.doi.org/10.20435/serie-estudos.v22i45.1072

\begin{abstract}
Resumen: La finalidad del presente artículo es analizar el proceso de privatización educacional chileno durante la Dictadura Cívico Militar (1973-1990), desde la publicación de la Directiva N 87 en enero de 1974 y la promulgación de la Ley Orgánica Constitucional de Enseñanza en marzo de 1990. En base al estudio de documentos relativos a la implementación del proceso de municipalización y descentralización de la educación en el sistema educativo chileno - en especial las Actas de las sesiones de la Junta Militar, del Consejo de Estado y de las Comisiones Legislativas que dieron lugar a la Ley Orgánica Constitucional de Enseñanza-, la investigación describe las líneas de acción y propuestas de diversos actores civiles y militares, y expone un análisis discursivo de las bases de la política educacional de la Dictadura Cívico Militar, considerando la introducción y consolidación del principio de subsidiariedad y la defensa de la libertad de enseñanza, pilares del sistema educacional nacional actual.
\end{abstract}

Palabras clave: libertad de enseñanza; principio de subsidiariedad; dictadura cívico militar.

Resumo: O objetivo deste artigo é analisar o processo de privatização durante o educativo CívicoMilitar ditadura chilena (1973-1990), desde a publicação da Directiva n. 87 em janeiro de 1974 e a promulgação da Lei Orgânica Constitucional de Educação em março de 1990. Com base no estudo de documentos relacionados com a implementação do processo de municipalização e descentralização da educação no sistema educacional chileno - especialmente as atas das reuniões da Junta Militar, o Conselho de Estado e das Comissões legislativas que gave -, o pesquisa descrever as linhas de ação e propostas de vários militares e atores civis e apresenta uma análise discursiva

\footnotetext{
${ }^{1}$ Pontificia Universidad Católica de Chile, Santiago, Chile.

2 Universidad Alberto Hurtado, Santiago, Chile.
} 
dos fundamentos da política educativa da Ditadura Civic Militar, considerando a introdução e consolidação do princípio da subsidiariedade e da defesa da liberdade de educação, pilares do atual sistema educativo nacional.

Palavras-chave: liberdade de educação; princípio da subsidiariedade; ditadura cívico-militar.

\begin{abstract}
The aim of this paper is to analyze the process of Chilean educational privatization during the Military-Civic Dictatorship (1973-1990), since the publication of Act No. 87 in January 1974 and the promulgation of the Organic Constitutional Law of Education in March 1990. Based on the study of documents related to the implementation of the process of municipalization and decentralization of education in the Chilean educational system-especially the Minutes of the meetings of the Military Junta, the Council of State and the Legislative Commissions that gave-, the research describes the lines of action and proposals of various civil and military actors and presents a discursive analysis of the bases of the educational policy of the Military Civic Dictatorship, considering the introduction and consolidation of the Principle of subsidiarity and the defense of Freedom of education, pillars of the current national educational system.
\end{abstract}

Key words: freedom of education; principle of subsidiarity; civil-military dictatorship.

\title{
1 LA ESTRATEGIA EDUCATIVA DE LOS CONTRAALMIRANTES: DIAGNÓSTICO, CAMBIO Y DESCENTRALIZACIÓN (1973-1976)
}

El Régimen Militar que siguió al Golpe de Estado de 1973 se orientó en base a dos referencias doctrinarias, las cuales se hacían presentes en todas las ocasiones en que los miembros del nuevo gobierno discutían sobre las políticas educacionales a implementar. Por una parte, las fuentes permiten develar y evidenciar la ideología de las Fuerzas Armadas-encarnadas en la Doctrina de la Seguridad Nacional ${ }^{3}$ - en el primer período de la Dictadura, y por otro, el liberalismo económico radical, representado por los herederos chilenos del economista estadounidense Milton Friedman, los Chicago Boys ${ }^{4}$.

En términos de evolución histórica, y con un Ministerio de Educación Pública dirigido por Contraalmirantes, el escenario educativo cambió radicalmente en septiembre de 1973: si desde la década de 1840 el Estado jugó un rol central con la promoción y orientación de la educación nacional, ya desde octubre de 1974 se evidencia un discurso pro descentralizador de la educación en los civiles ${ }^{5}$ que

\footnotetext{
${ }^{3}$ Al respecto, es conveniente revisar las publicaciones periódicas de la Academia Superior de Seguridad Nacional, tituladas Seguridad Nacional (1977).

${ }^{4}$ Grupo de economistas chilenos que realizaron estudios de postgrado en la Escuela de Economía de la Universidad de Chicago en las décadas de 1960 y 1970, y que asumieron funciones gubernamentales en la Dictadura del General Augusto Pinochet.

${ }^{5}$ Los civiles que trabajaban para la Oficina de Planificación Nacional (ODEPLAN) promovieron insis-
} 
asesoraban a los miembros de la Junta Militar. Éstos diagnosticaban la crisis social como una consecuencia natural de la fuerte injerencia del Estado en el área social, en cuanto limitaba la acción particular y no distribuía eficientemente los recursos: "Uno de los problemas más graves que tenemos en Chile es el sistema educacional que hemos seguido tradicionalmente" (JUNTA DE GOBIERNO, 1974, p. 12).

Consecuentemente con la Doctrina de Seguridad Nacional, la estrategia educativa de los Contraalmirantes estuvo orientada por tres tópicos principales: Seguridad, Desarrollo y Libertad (ACADEMIA SUPERIOR DE SEGURIDAD NACIONAL, 1977). Si bien a nivel de la micropolítica y de la cotidianeidad de la escuela los cambios fueron radicales, autoritarios y drásticos una vez consumado el Golpe de Estado (PÉREZ NAVARRO, en prensa), a nivel institucional los cambios fueron paulatinos y planificados, ya que los Contraalmirantes no obedecieron inmediatamente a las presiones de grupos oligárquicos a favor de una liberalización absoluta de la enseñanza. Al respecto, es fundamental señalar lo establecido por la Directiva $N^{\circ}$ 87 (MINISTERIO DE EDUCACIÓN PÚBLICA, 1973), uno de los primeros documentos que exponían las orientaciones educativas a seguir, que fue socializada por el Régimen a través del periódico El Mercurio (1974, p. 30). De acuerdo a lo señalado por Santiago Quer Antich, académico de la Escuela de Educación de la Universidad Católica de Chile, la Directiva reforzaba el principio centralista del Estado en materia educativa, en cuanto presentaba al Ministerio de Educación Pública como el organismo coordinador y regulador de todas las instituciones educacionales, incluso los colegios y universidades particulares. De esta forma, quedaba en evidencia la primera estrategia educativa de los Contraalmirantes de controlar completamente el sistema de educación nacional:

La 'Directiva' está inserta, a nuestro juicio, en un esquema y concepción estadodocentista de la Educación, consecuente con lo cual, ve deprimida la Universidad su autonomía y se compartimentiza de acuerdo con los intereses del desarrollo de la nación que tenga el equipo gobernante que determine la línea educacional. (QUER, 1974).

No obstante, la Directiva ya señalaba los pasos que seguirían: mientras se terminaba con la amenaza marxista al interior del sistema educativo, se planificaba la descentralización de las funciones del Ministerio de Educación Pública. A través de dicho documento, se ordenaba la organización de comisiones de trabajo para

tentemente una política de descentralización para todas las áreas de la administración pública. 
el diagnóstico del estado de la educación chilena, con los objetivos de evaluar los efectos de la implementación de la Reforma Educacional de 1965, "determinar la correspondencia de la educación con las necesidades globales y específicas del país $y$, al mismo tiempo, plantear dentro de dicho marco las pautas y estrategias que consoliden una política integral de la educación acorde con las demandas del desarrollo nacional y regional" (MINISTERIO DE EDUCACIÓN PÚBLICA, 1974). La realización de la Consulta a nivel de unidad educativa se inició en enero 1974, con la organización de 30 comisiones de estudio, integradas por más de 400 personas.

Los resultados del diagnóstico, difundidos a través de los periódicos La Tercera y El Mercurio en el mes de julio, convencieron a las autoridades militares sobre la necesidad de embarcarse en una reorientación de la educación nacional (SUPERINTENDENCIA DE EDUCACIÓN, 1975). En las sesiones de la Junta de Gobierno, el gobierno de facto señalaba la necesidad reorganizar el sistema, puesto que el Estado-a través del Ministerio de Educación- se hacía cargo de tres millones de estudiantes, y de los múltiples problemas que esto conllevaba. Asimismo, la decisión de descentralizar la administración pública -y la responsabilidad educacional del Estado- fue justificada por las autoridades militares considerando la 'realidad anárquica' que reinaba en los distintos ministerios y servicios públicos con anterioridad al 'Pronunciamiento militar'. Así fue señalado por el General Pinochet en el discurso de conmemoración del primer aniversario del 11 de septiembre:

Tanto el Ministerio como cada uno de sus Servicios mostraban un alto grado de desorganización, ineficiencia y anarquía estructural. Proliferaban las jefaturas, oficinas y funcionarios innecesarios que sólo cumplían, a menudo, funciones estrictamente políticas. (CHILE, 1974).

La solución a la crisis social y educativa diagnosticada por las Fuerzas Armadas fue la descentralización de los servicios educacionales (opinión compartida por la mayoría de los civiles designados en las distintas instituciones estatales). En una sesión secreta celebrada por la Junta Militar el primer día de octubre de 1974, un funcionario de ODEPLAN-invitado como asesor- exponía los cambios que los técnicos y especialistas estaban barajando para delegar funciones ministeriales a actores e instituciones intermedias:

En este sentido estamos preparando algún material para discutirlo con los Ministerios y proponer algunos mecanismos que permitan una llegada efectiva. Por ejemplo, en alimentación escolar, creemos que el mecanismo debería ser más a través de las provincias y municipalidades, una asignación directa de un vale que dé derecho a una participación. Con ello, el individuo que estaría reci- 
biendo este derecho podría hacer uso de ese vale en cualquiera institución, sea estatal o privada, siempre que esté dentro de los marcos de la extrema pobreza [...] Entonces, ahí estamos explorando la posibilidad de darle una especie de vale o cheque educativo a cierta gente que nos interesa, que puede utilizarlo en alternativas previamente aprobadas por el Ministerio de Educación, sean escuelas estatales y privadas. Esta es toda una línea que estamos tratando de explorar y estamos preparando algunos documentos para discutirlos con cada uno de los Ministerios una vez que llegue el momento, a ver qué les parece a ellos y asegurar que se diseñe un programa que permita llegar a los sectores de extrema pobreza. (JUNTA NACIONAL DE GOBIERNO, 1974, p. 8).

El proceso de regionalización, implementado por la Comisión Nacional de Reforma Administrativa (CONARA) a lo largo de 1974, se convirtió en el mecanismo por excelencia para terminar con las deficiencias que traía consigo la concentración de tareas del Estado y, de paso, terminar con la histórica y, a su modo de ver, anacrónica figura del Estado Docente:

La Administración de la Educación se inscribe dentro del marco de la reforma integral de la administración del Estado, cuyos objetivos más importantes, en lo que atañe a educación, son:

a. La desburocratización integral de la administración.

b. La redefinición y el perfeccionamiento del sistema de administración del personal.

c. El afianzamiento del proceso de regionalización, el fortalecimiento del rol del Alcalde y el mejoramiento de las Municipalidades como organismos de participación y de movilización de la comunidad y de prestación de servicios.

d. La revisión y consolidación de la reestructuración general de los ministerios, servicios e instituciones públicas. (OFICINA DE RELACIONES INTERNACIONALES, 1981, p. 13).

Justificada en base a la Doctrina de Seguridad Nacional, la descentralización de los servicios educacionales permitiría al Estado un crecimiento integral, en cuanto evitaría la existencia de zonas territorialmente alejadas, deprimidas y administrativamente desvinculadas del conjunto "más uniforme, y que puedan presentarse como puntos vulnerables en términos de Seguridad Nacional" (ACADEMIA SUPERIOR DE SEGURIDAD NACIONAL, 1976). Los fundamentos de la relación entre los cambios descentralizadores en educación y la regionalización administrativa del país se pueden observar en el siguiente párrafo, extraído de la revista de la Academia Superior de Seguridad Nacional, institución docente del Ministerio de Defensa:

Como la búsqueda del crecimiento integral ya no es posible llevarla al plano del centralismo que hasta el presente hemos padecido, adquiere relevancia 
la idea moderna del desarrollo regional y su fenómeno administrativo que es la regionalización. (ACADEMIA SUPERIOR DE SEGURIDAD NACIONAL, 1976).

Poco tiempo después del Golpe Militar la descentralización de los servicios educacionales pasó a ser una realidad. A partir del diagnóstico de la situación educativa del país, las autoridades militares definieron los principios que sustentarían la política educacional que comenzaría a ser aplicada. De acuerdo al Plan Operativo Nacional y Regional del Sector Educación de 1975, los cambios estarían orientados por los siguientes lineamientos: renovación continua, cambio planificado, pluralismo y educación permanente. El análisis del documento, publicado por la Superintendencia de Educación Pública, exponía que las políticas a implementar no tenían carácter de reforma o transformación radical del sistema educativo, sino que éste se renovaría paulatina y continuamente:

[...] la adaptación de la educación a la realidad nacional y a los anhelos de los individuos de esta sociedad es una obra continua. No se plantea una nueva 'reforma' sino que la renovación continua es la modalidad organizadora de la Educación. (SUPERINTENDENCIA DE EDUCACIÓN, 1975, p. 1).

La renovación se haría de manera planificada, mediante una consulta amplia a distintos sectores, y con una evaluación rigurosa de los objetivos logrados. Asimismo, y consecuente con la idea del Estado Subsidiario definida en la Declaración de principios del Gobierno de Chile difundida por la Junta Militar en 1974, se reconoció la función educativa de la sociedad en su conjunto, ante lo cual se señalaba que el Ministerio de Educación tendría solo el control necesario para la protección del interés público.

Si 1974 fue el año de la elaboración de la política educacional del Régimen, 1975 fue el período de ejecución e implementación de los proyectos de descentralización. Así lo aseguraba Pinochet en 1975:

Para dar cumplimiento a la política de descentralización y desconcentración de los Servicios, el Ministerio ha desarrollado en forma prioritaria el plan de regionalización. Se encuentran en funcionamiento las 12 Secretarías Regionales de Educación, de las cuales 5 son Secretarías Ministeriales y está en estudio final la organización del Área Metropolitana. (CHILE, 1974, p. 385).

De esta forma, entre los años 1973 y 1976 se consolidó la primera etapa de descentralización de la educación nacional. Lentamente, el Estado Docente fue siendo sepultado por la Reforma Administrativa implementada por CONARA, y la Doctrina de Seguridad Nacional dio paso a la introducción de principios neoliberales en materia educacional. Pero, ¿cómo se produjo el cambio? ¿Por qué terminó predominando 
Estado Docente, subsidiariedad y libertad de enseñanza. El proceso de privatización educacional en Chile desde la perspectiva de los actores (1973-1990)

la ideología de los colaboradores civiles en lugar de los elementos doctrinarios de las Fuerzas Armadas?

\section{DE LA DESCENTRALIZACIÓN MINISTERIAL A LAS ESCUELAS MUNICIPALIZADAS: DISCURSOS Y ACTORES EN EL PROCESO DE PRIVATIZACIÓN EDUCACIONAL}

La definición y discusión sobre los cambios a introducir en materia educacional generó que los actores involucrados en la administración del Régimen se posicionaran y defendieran perspectivas distintas. En un contexto de fuerte represión e implementación de medidas dictatoriales, la deliberación respecto a la política educacional sólo podía darse al interior de la Junta Militar, y por actores civiles legitimados por las autoridades militares.

\section{JUNTA MILITAR: PRINCIPIO DE SUBSIDIARIEDAD Y EDUCACIÓN}

Desde la Declaración de Principios del Gobierno de Chile, elaborado en 1973 y publicado en 1974 por la Junta de Gobierno, el principio de subsidiariedad del Estado se presentó como una orientación prioritaria en el discurso oficial, vinculándose directamente a la nueva concepción filosófica del hombre y de la sociedad que se intentaba difundir. Al parecer de la Junta, al ser el hombre-y sus derechos naturales- anteriores y superiores al Estado, éste debería estar al servicio del hombre, y no al revés:

[al Estado] le corresponde asumir directamente sólo aquellas funciones que las sociedades intermedias o particulares no están en condiciones de cumplir adecuadamente [...] Respecto al resto de las funciones sociales, sólo puede entrar a ejercerlas directamente cuando las sociedades intermedias que 'de suyo 'estarían en condiciones de asumirlas convenientemente, 'por negligencia o fallas' no lo hacen, después 'de que el Estado haya adoptado las medidas para colaborar a que esas deficiencias sean superadas'. En este caso, el Estado actúa 'en subsidio' por razón del bien común. (JUNTA NACIONAL DE GOBIERNO, 1974, p. 4).

Por otro lado, en 1975 la Junta Militar publicó y mandó a difundir un documento con las orientaciones futuras del Régimen, el Objetivo Nacional del Gobierno de Chile, el cual tenía como principal finalidad orientar hacia objetivos comunes "tanto las acciones específicas como sectoriales que cumplirá realizar a los Organismos de Estado, en procura de alcanzar aquellas metas de bien común" (JUNTA DE GOBIERNO, 1975 , p. 16). En este texto se determinaba que, para cumplir con la finalidad de 
profundizar y transmitir el amor a la Patria, el respeto a la vocación libre y transcendente del ser humano, el aprecio por la familia como célula básica y la adhesión al concepto de unidad nacional, el Estado

reconoce el derecho preferente de los padres a educar a sus hijos, y admite una amplia libertad de enseñanza, sin otros límites que los que el bien común impone a la función educacional [...]

El Estado considera como una de sus funciones más esenciales, la de asegurar que la enseñanza básica obligatoria sea una realidad para todos los chilenos, proporcionándosela gratuitamente al menos a quienes no puedan subvenir económicamente a ella. (JUNTA DE GOBIERNO, 1975).

\section{LA COMISIÓN ‘ORTÚZAR’: DEBATE Y PROPUESTA DE LAS GARANTÍAS CONSTITUCIONALES}

El día de 23 de junio de 1975 la Comisión Ortúzar acordó iniciar el estudio de la garantía constitucional de la Libertad de Enseñanza. Para esto invitaron a las sesiones a distintas personas relacionadas con el sistema educativo, con el objetivo de escuchar opiniones especializadas. El 10 de Julio, luego de 17 días de exposiciones relativas al tema, la Comisión comenzó a discutir la garantía relativa que la futura Constitución debía otorgarle a la Libertad de Enseñanza.

La garantía que la Constitución de 1925, vigente hasta ese entonces, le otorgaba a la Libertad de Enseñanza fue un punto de divergencias entre los integrantes de la Comisión. Por un lado, algunos señalaban que dicho texto constitucional aseguraba en lo esencial la Libertad de Enseñanza. A raíz de una proposición tentativa que el padre Eugenio León Bourgeoi, vocero del Episcopado de Chile, envió a la Comisión ${ }^{6}$, el señor Alejandro Silva Bascuñán señaló que el precepto vigente había servido a todos para defender a Libertad de Enseñanza. Incluso, dicho precepto legal sirvió para manifestar que los ideales contenidos en la ENU eran absolutamente contrarios a la Constitución y más cercanos a los ideales de "traición a la democracia y la historia chilena que inspiraban a los que estaban gobernando" (COMISIÓN DE ESTUDIOS DE LA NUEVA CONSTITUCIÓN POLÍTICA DE LA REPÚBLICA DE CHILE, 1975, p. 101).

\footnotetext{
${ }^{6}$ En 1975 el Departamento Educacional de Episcopado Chileno envió a los Obispos y presidentes de las oficinas diocesanas de educación católica un documento que contenía diversas apreciaciones de la Iglesia sobre la Libertad de Enseñanza. Dicho documento fue enviado a la Comisión Ortúzar, que estudiaba en paralelo las futuras reformas constitucionales, para que ésta considerara y ponderara los planteamientos de la Iglesia Católica sobre la garantía constitucional. Ver: Editorial El Mercurio, Santiago, Chile. 1 de Julio de 1975.
} 
Estado Docente, subsidiariedad y libertad de enseñanza. El proceso de privatización educacional en Chile desde la perspectiva de los actores (1973-1990)

Esta defensa de la garantía constitucional de la Libertad de Enseñanza por parte de la Constitución de 1925 encontró oposición en otro integrante, el señor Sergio Díez Urzúa, quien manifestó considerar insuficiente el resguardo que el texto constitucional vigente le otorgaba a la Libertad de Enseñanza, ya que él mismo, cuando era presidente de la Comisión de Educación del Senado, tuvo que enfrentarse con el Ministerio de Educación cuando éste intentó mantener una autoridad absoluta sobre la Superintendencia de Educación a través de una interpretación torcida y sinuosa de la Constitución de $1925^{7}$. En consecuencia, Díez Urzúa consideraba que el texto constitucional de 1925 conducía inevitablemente a confusiones y las malas interpretaciones en materias fundamentales, especialmente en relación al concepto de Libertad de Enseñanza.

La discusión en torno a este derecho suscitada en las sesiones de la Comisión Ortúzar se caracterizó por un marcado pragmatismo, principalmente porque los actores involucrados evidenciaban el problema técnico que la Comisión debía afrontar al garantizar constitucionalmente tal derecho, en perjuicio de un debate más profundo que abordara la concepción y el significado de la Libertad de Enseñanza. Al respecto, Díez Urzúa sostenía que:

[...] el problema es más técnicamente delicado que filosóficamente delicado. Filosóficamente, los miembros de esta Comisión tienen una idea clara de lo que es Libertad de Enseñanza, y las divergencias pueden surgir respecto de la técnica legislativa adecuada para asegurar la Libertad de Enseñanza y, al mismo tiempo, para garantizar que la enseñanza libre cumpla con los objetivos fundamentales que una sociedad bien constituida debe pretender que ella alcance. (COMISIÓN DE ESTUDIOS DE LA NUEVA CONSTITUCIÓN POLÍTICA DE LA REPÚBLICA DE CHILE, 1975, p. 108).

A pesar de que la Comisión concebía a la Libertad de Enseñanza una garantía fundamental y de vital importancia para el país, ya que implicaba "la formación de las generaciones futuras", los integrantes sostenían que ésta por sí sola no era suficiente para asegurar el correcto funcionamiento del sistema educacional. Así pues, proponían articular esta garantía con el principio de subsidiariedad, principio rector de la Dictadura Militar, el que a su parecer debía estar explícitamente consagrado en la Constitución. De esta manera, la educación privada no se pensaba

\footnotetext{
${ }^{7}$ Sergio Díez hacía referencia al período 1972- 1973, cuando él era diputado por Linares, Loncomilla y Parral. Es preciso recordar que en ese tiempo se intensificó el debate sobre Libertad de Enseñanza, principalmente por la discusión generada por el proyecto de Escuela Nacional Unificada (ENU).
} 
como cooperadora de la responsabilidad educacional del Estado, sino que, por el contrario, el Estado debía ser cooperador de la educación particular, tal como lo sugirió Díez Urzúa:

Asimismo, debe consagrarse en la Constitución el principio de que la sociedad debe colaborar al funcionamiento de la educación privada. No se trata sólo de garantizar la libertad de enseñanza, por cuanto ello no es suficiente para un país como Chile. Eso es una declaración teórica que no tiene más efectos que los que le señale la ley, como decía don Enrique Evans, pero que efectivamente no produce como consecuencia una garantía de libertad de enseñanza si no se establece la obligación del Estado de concurrir a la subvención, financiamiento o mantenimiento de los establecimientos privados de educación que cumplan, evidentemente, con las normas mínimas que el mismo Estado les fije. (COMISIÓN DE ESTUDIOS DE LA NUEVA CONSTITUCIÓN POLÍTICA DE LA REPÚBLICA DE CHILE, 1975, p. 109).

Sin embargo, estas subvenciones no debían restringirse bajo ningún precepto que no fuera la búsqueda de fines de lucro. De acuerdo a algunos miembros de la comisión, limitar o condicionar el aporte del Estado era considerado una directa vulneración al principio de subsidiariedad. Por otro lado, también se advertía que el fin de lucro tergiversaría y convertiría la educación en una empresa comercial:

[...] hay en la Constitución actual una cosa que es profundamente errónea y perturbadora: sólo la educación privada gratuita y que no persigue fines de lucro recibe una contribución. No. La que debe recibir una contribución del Estado es toda educación que no persiga fines de lucro, sea gratuita o no, porque la gratuidad va a depender del acuerdo de los padres, del grado de educación que se dé en la escuela y del grado de eficiencia de los profesores. Lo que se necesita, para recibir la subvención del Estado, es que la finalidad sea educar y nada más que educar, y no transformar la educación en una empresa comercial. (COMISIÓN DE ESTUDIOS DE LA NUEVA CONSTITUCIÓN POLÍTICA DE LA REPÚBLICA DE CHILE, 1975, p. 109).

El discurso de la Comisión Ortúzar sobre la Libertad de Enseñanza fue, entonces, un discurso pragmático, que entregaba referencias mayormente a la concepción ontológica de dicha garantía, y se limitaba a discutir los preceptos jurídicos más adecuados para asegurarla. Además, es posible apreciar-a pesar de la existencia de elementos de liberalización del sistema educativo- cierta aprehensión hacia la mercantilización de la educación, en cuanto se rechazaba explícitamente (por parte de algunos miembros de la Comisión) la búsqueda de lucro en el sistema educacional. 


\section{CONSEJO DE ESTADO: DERECHO A LA EDUCACIÓN Y LIBERTAD DE ENSEÑANZA}

El Consejo de Estado, al debatir sobre el Derecho a la Educación y la Libertad de Enseñanza, buscaba "promover la formación de la juventud y del pueblo, sobre la base de ciertos principios esenciales que son los que precisamente los enemigos de la libertad y de la democracia tratan de destruir" (CONSEJO DE ESTADO, 1978, p. 635).

La Comisión dedicó gran parte del tiempo de las sesiones a un prolongado debate sobre si el Estado, en su papel de garante del bien común, debía tener la atención preferente de la educación, o, por el contrario, si la Constitución debía explicitar que los padres, en tanto son, según el principio de subsidiariedad, anteriores y superiores al Estado, tienen la atención prioritaria de educar a sus hijos.

Dos grupos estuvieron claramente enfrentados. En el primer grupo se sostenía que el principio de subsidiariedad no era suficiente argumento como para desligar del Estado la atención preferente de la Educación. Sobre esto, se declaraba que:

[...] el principio de subsidiaridad no es aplicable ni a la salud pública ni a la educación. [...] ese concepto ha sido forjado por los economistas para los efectos de motivar la iniciativa privada en la creación de riqueza y de fuentes de trabajo para elevar el nivel de vida; pero que la salud y la educación pública no pueden ser sino responsabilidad del estado, por cuanto éste es protagonista del interés nacional. (CONSEJO DE ESTADO, 1978, p. 638).

El presidente del Consejo de Estado, el ex presidente Jorge Alessandri Rodríguez, también se encontraba dentro de este primer grupo, ya que, a su entender, explicitar que el Estado tiene la atención preferente de la educación, tal como lo señala la Constitución de 1925, le había importado al Fisco considerables ingresos económicos (CONSEJO DE ESTADO, 1979, p. 643), posición que compartía el también ex presidente de la República, don Gabriel González Videla. En consecuencia, Alessandri Rodríguez dejó constancia en el Consejo de que "lamenta profundamente que no se haya considerado que al Estado corresponda la supervisión de todas las actividades educacionales" (CONSEJO DE ESTADO, 1979, p. 649). Sin embargo, evitó formular indicación porque no deseaba provocar conflictos en el seno de la Comisión, a la vez que advertía que:

[...] gran parte del siglo pasado en Chile, se caracterizó por la lucha desarrollada para dar supremacía al Estado en materia educacional, por lo que puede ser peligroso retrotraer la discusión a esa época, permitiéndose, tal vez que se califique de reaccionario un texto que omite la supervisión del Estado. (CONSEJO DE ESTADO, 1979, p. 649). 
Por el contrario, en el segundo grupo se argüía que recaía en los padres la educación de sus hijos ya que el principio de subsidiariedad así lo establecía. Al respecto, Carlos Cáceres Contreras advertía que el Consejo debía analizar la educación remitiéndose obligadamente al principio de subsidiariedad, según el cual "la responsabilidad en el proceso educativo corresponde, en primer lugar, a los padres de familia, al núcleo familiar, y, en segundo término, a las entidades privadas" (CONSEJO DE ESTADO, 1978, p. 638). En consecuencia, a su modo de ver, el rol del Estado en educación se debería restringir a las siguientes tareas:

[...] primera, cuidar de que el proceso educacional no atente contra el bien común; segunda, exigir el cumplimiento de ciertos requisitos mínimos de enseñanza, en materia de programas, de profundidad, de contenido de los mismos; $y$, tercera, financiar el proceso educacional en el subsidio de la familia y de las entidades privadas, cuando éstas no tengan posibilidades de hacerlo. (CONSEJO DE ESTADO, 1978, p. 638).

Finalmente, el Consejo de Estado estimó conveniente dejar intacta la propuesta sobre el derecho a la educación de la Comisión redactora del anteproyecto constitucional, que decía lo siguiente:

9.- El derecho a la educación.

La educación tiene por objeto el pleno desarrollo de la persona en las distintas etapas de su vida.

La educación básica es obligatoria. El estado deberá mantener un sistema gratuito con tal objeto.

Le corresponderá, asimismo, fomentar el desarrollo de la educación en todos sus otros niveles; estimular la investigación científica y tecnológica, la creación artística y la protección e incremento del patrimonio cultural de la nación. Los padres tienen el derecho y el deber de educar a sus hijos" (CONSEJO DE ESTADO, 1979, p. 647).

\section{COMISIÓN CONJUNTA Y EL PROYECTO DE LEY ORGÁNICA}

Los integrantes de las sesiones de la Comisión Conjunta fueron especialmente meticulosos en que las normas redactadas en la Ley Orgánica Constitucional de Enseñanza no vulneraran las libertades garantizadas por la Constitución de 1980, especialmente la relativa a la Libertad de Enseñanza. De esta manera, la Libertad de Enseñanza se presentaba en la discusión sobre la Ley como un principio doctrinario, el cual era respetado por todos los integrantes a la hora de hacer algún comentario.

A su vez, los integrantes constantemente abogaban porque los establecimientos educacionales tuvieran una amplia libertad para elaborar sus propios planes y 
Estado Docente, subsidiariedad y libertad de enseñanza. El proceso de privatización educacional en Chile desde la perspectiva de los actores (1973-1990)

programas, que el Estado desarrollara planes y programas propios para los establecimientos municipales ${ }^{8}$, y que cualquiera pueda optar por ellos. Como justificación para tal posición, y a modo de legitimación jurídica, los integrantes invocaban sistemáticamente la Libertad de Enseñanza. Al respecto, la secretaria de la Comisión, señora Eugenia Ramírez Faúndez, señalaba:

La Comisión Conjunta, partiendo de la Libertad de Enseñanza, estima que cada establecimiento puede determinar sus programas educacionales, ya sea por etapas o niveles a través de los contenidos mínimos y no a través de programas que podrían anular dicha libertad. (SESIONES CONJUNTAS DE LAS COMISIONES LEGISLATIVAS, 1988).

De esta manera no solo se pregonaba por la máxima libertad para establecer planes y programas de estudio, sino que también se buscaba reducir la influencia de los planes de estudio desarrollados por el Estado, ya que, a juicio de los integrantes de la Comisión Conjunta, imponer sistemas de enseñanza atentaba directamente contra la libertad garantizada constitucionalmente. A diferencia de las antiguas Constituciones políticas, en las que se garantizaba la Libertad de Enseñanza, pero, a su vez, se decretaba que todas las instituciones educativas debían ajustarse "a los planes y programas establecidos por las autoridades educacionales" (MINISTERIO DEL INTERIOR, 1925), la Constitución de 1980 implanta, al amparo de la Libertad de Enseñanza, una libertad total de sostenedores y autoridades educacionales para elaborar planes y programas.

La garantía que la Libertad de Enseñanza le otorga al derecho de abrir, organizar y mantener establecimientos educacionales es un tema que devela cierta contradicción ideológica entre los miembros de la Comisión Conjunta: por un lado defendían aferradamente la libertad para abrir establecimientos educacionales y demostraban gran aprensión cada vez que, a su juicio, se vulneraba tal derecho; y por otro lado, rechazaban el hecho de que proliferaran establecimientos de educación superior que no cumplían con las condiciones materiales ni pedagógicas necesarias para impartir educación, por lo que proponían que el sistema de acreditación de instituciones de educación regulara la normativa al respecto:

\footnotetext{
${ }^{8}$ Cabe señalar que existe una confusión entre los integrantes, excepto en los que están relacionados profesionalmente con el sistema educacional, en calificar a los establecimientos como privados o, en su defecto, fiscales, siendo que la dependencia administrativa de los establecimientos educacionales escolares permite calificarlos en: particulares pagados, particulares subvencionados y municipalizados. Es, precisamente, a estos últimos que los integrantes se refieren incorrectamente como fiscales.
} 
En otras palabras, bien es que haya absoluta libertad para abrir estos establecimientos, pero bajo ciertas condiciones mínimas. Porque hoy día es una realidad que hay ciertas instituciones que tiene sólo el nombre de universidades, dado que están cubriendo un aspecto mínimo de lo que constituye una universidad y, a veces, sin la más elemental infraestructura como para impartir una docencia a nivel superior con seriedad y con responsabilidad. (SESIONES CONJUNTAS DE LAS COMISIONES LEGISLATIVAS, 1989).

Por un lado se buscaba ampliar las facultades y libertades para abrir y organizar colegios, intención acorde con el modelo económico neoliberal que impuso la dictadura, pero, por otro lado, intentaban que se regularizara tal libertad definiendo las condiciones mínimas que demandaba crear una institución de educación superior, propugnado así que el Estado tenga una participación más activa en el tema. A su vez, los integrantes pertenecientes a las fuerzas armadas se mostraban reacios a dejar al Estado sin ninguna forma de regulación del sistema educativo. Lo anterior reafirma lo sostenido sobre que en la estructura educacional del régimen militar convivían dos formas distintas de concebir el sistema educativo: una técnico economicista, cercana a los Chicago Boys, marcada por una lógica de mercantilización y liberalización, y otra representada por las autoridades militares, que pretendían mantener, mediante una renovación continua, el desarrollo lineal del sistema educativo (PROGRAMA INTERDISCIPLINARIO DE INVESTIGACIONES EN EDUCACIÓN [PIIE], 1984).

Cualquier tipo de regulación o participación del Estado en educación era considerada en el discurso de los integrantes de la Comisión Conjunta como una coartación del derecho constitucional a la Libertad de Enseñanza. Así pues, no solo eran consideradas limitaciones a la Libertad de Enseñanza el atentar contra la moral, las buenas costumbres, el orden público y la seguridad nacional, sino que también la imposición de planes y programas desarrollados por el Estado a establecimientos educacionales que no eran públicos (SESIONES CONJUNTAS DE LAS COMISIONES LEGISLATIVAS, 1989), la formulación de contenidos obligatorios de aprendizajes según niveles de enseñanza y las normativas que regulen la creación de instituciones educativas (SESIONES CONJUNTAS DE LAS COMISIONES LEGISLATIVAS, 1989). La vulneración del derecho a la Libertad de Enseñanza era evocada por los integrantes de la Comisión Conjunta para deslegitimar cualquier indicación, norma legal y decisión política que pretendiera regular, fiscalizar o normar el campo educativo.

Al mismo tiempo, el principio de Libertad de Enseñanza y sus limitaciones explicitadas en la Constitución de 1980 era constantemente instrumentalizada por los integrantes de la Comisión Conjunta para otorgar un fundamento jurídico a su decisión 
de incorporar un representante de las fuerzas armadas y carabineros en el Consejo Superior de Educación, organismo público y autónomo creado por la Ley Orgánica Constitucional de Enseñanza y cuyo objetivo era asegurar la calidad de la educación. Se evidencia así que los integrantes de la Comisión pertenecientes a alguna rama de las fuerzas armadas ejercieron presión y utilizaron resquicios legales para satisfacer sus intereses corporativos. Esto refuerza lo planteado anteriormente, sobre que la integración de la Comisión Conjunta no obedecía solo a criterios educativos, sino también a intereses políticos y gremiales.

Es importante señalar que a pesar de preconizar constantemente la libertad, algunos miembros también rechazaban lo que ellos mismos denominan "libertinaje", que sería la libertad exacerbada, la libertad que en su ejercicio, paradojalmente, atentaba contra la Libertad de Enseñanza, tal como sostenía Gabriel Ogalde: "La posición de la Cuarta Comisión es una posición de consagrar una amplia libertad, con los resguardos necesarios y estrictamente precisos para que no degenere en libertinaje y así proteger al educando". El presidente de la Comisión Conjunta, General Montero Marx, era más elocuente al respecto: "[...] y así evitar que esta libertad se convierta en un libertinaje que nos lleve el día de mañana a un verdadero desastre" (SESIONES CONJUNTAS DE LAS COMISIONES LEGISLATIVAS, 1988).

Por otro lado, los integrantes de la Comisión Conjunta estaban conscientes de que en las antiguas Constituciones el Estado debía dar atención preferente al Derecho a la educación, pero ahora, en consonancia con su rol subsidiario, debía limitarse a velar por calidad de la educación entregada por los particulares y a entregar educación allí donde los particulares no estaban interesados en hacerlo. Este cambio rupturista de un Estado Intervencionista en educación, como lo consagra la Constitución de 1925, a uno Subsidiario, se explica en gran parte por la sistemática utilización de la Libertad de Enseñanza como premisa ideológica por parte de los agentes del campo educativo, entre los que se encontraban los integrantes de la Comisión Conjunta.

El principio de subsidiariedad del Estado también fue un tema recurrente en las sesiones de la Comisión Conjunta. En efecto, los integrantes estaban convencidos del rol subsidiario del Estado en educación y no había mayores discrepancias en el seno de la Comisión al respecto. Como declaraba Montero Marx: "Bien, el quinto punto sería el papel del Estado, el cual yo subdivido en un papel definitivamente subsidiario en el desarrollo mismo de la educación" (SESIONES CONJUNTAS DE LAS COMISIONES LEGISLATIVAS, 1988). 
Las atribuciones que la Comisión Conjunta le asignaba al Estado en su rol subsidiario se pueden dividir en básicamente dos funciones: subsidiar la educación particular y entregar apoyo técnico a los establecimientos que lo necesiten:

$\mathrm{Si}$ el Estado subvenciona es porque en aquellas materias-cualquiera que sea, ya sean educacionales u otras- los particulares no pueden realizar por sí solos. Es decir, cuando los particulares no pueden realizar o acotar cierta actividad el Estado tiene dos maneras de actuar: haciéndolo directamente a través de sus órganos o pagándole una subvención a los particulares. (SESIONES CONJUNTAS DE LAS COMISIONES LEGISLATIVAS, 1988).

Por tanto, para los integrantes de la Comisión el rol subsidiario del Estado implicaba que éste debiera atender preferentemente los establecimientos particulares, en cuanto la educación es una función que los particulares sí estaban en condiciones de atender adecuadamente, y, en última instancia, velar por la educación pública. Así pues, en función del principio de subsidiariedad, Ramón Suárez enumeraba las funciones del Estado en educación de la siguiente manera:

[...] que de alguna manera quedara establecido el rol subsidiario del Estado por las etapas que corresponde. Es decir, el primer rol es orientador de la educación que los privados desarrollan. Si acaso no hay privados que desarrollen está toda la acción del Estado a través del subsidio a los particulares. Si no hay particulares interesados en visualizar esto a través de los subsidios, antes de eso está el subsidio a los colegios municipales, el apoyo a la municipalización y, por último, está el rol directo de crear establecimientos privados. (SESIONES CONJUNTAS DE LAS COMISIONES LEGISLATIVAS, 1988).

De esta forma, el principio de subsidiariedad del Estado atentó directamente contra la educación pública, relegándola a un segundo plano respecto a la educación privada. Así pues, Libertad de Enseñanza y principio de subsidiariedad fueron instrumentalizados de manera articulada por los integrantes de la Comisión Conjunta para reducir al mínimo posible la intervención del Estado en educación.

El hecho de reducir la participación del Estado en educación-y en todos los aspectos económicos y sociales-, aparece, entonces, en el discurso de los integrantes como el verdadero y último objetivo del régimen militar:

Por eso pienso-conversando en borrador-, sustentando el principio de libertad -del que yo participo y del que participa todo este Gobierno, porque para eso se hizo el 11 de septiembre-, pienso que la intervención del Estado debe ser mínima y muy claramente establecida; de otra manera nos vamos a encontrar, como muy bien lo dijo el señor Lecaros, con esto de los programas, los planes 
Estado Docente, subsidiariedad y libertad de enseñanza. El proceso de privatización educacional en Chile desde la perspectiva de los actores (1973-1990)

y estudios obligatorios, y vamos a estar metidos en una nueva ENU. (SESIONES CONJUNTAS DE LAS COMISIONES LEGISLATIVAS, 1987).

\section{CONCLUSIONES}

El análisis de las sesiones de la Junta Militar, el Consejo de Estado, la Comisión Ortúzar y la Comisión Conjunta permite observar las divergencias en materia de educación existente entre los dos grupos que se hicieron cargo del país luego del Golpe de Estado, divergencias que tuvieron efectos directos en la manera de concebir y regular el sistema educativo. Por un lado, los integrantes de las Fuerzas Armadas, representados por los Contralmirantes que dirigieron el Ministerio de Educación, sostuvieron la necesidad de introducir cambios paulatinos en el sector educación, atendiendo a los conceptos de renovación continua y planificación. Por otro lado, es posible evidenciar a un grupo de civiles, los Chicago Boys, quienes defendían y propugnaban la aplicación de principios economicistas y mercantilistas en la educación, basados en un profundo neoliberalismo económico.

Si bien esta tensión entre ambos grupos pareció resolverse a comienzos del régimen militar a favor del discurso de los integrantes de las fuerzas armadas, que pregonaba una mantención del Estado como ente regulador y coordinador en materia educativa, finalmente se decantó por el discurso neoliberal defendido por los civiles colaboradores, los denominados Chicago Boys. De esta forma, la resolución de dicha tensión a favor del discurso neoliberal en detrimento del discurso mantenido por los militares permitió que principios de liberalización y mercantilización se introdujeran y consolidaran en el sistema educacional. Las fuentes revelan que esta preeminencia de la ideología neoliberal sólo fue posible gracias a la estrategia empleada por el sector civil del gobierno de facto de instrumentalizar principios como la Libertad de Enseñanza y el de Subsidiariedad.

Finalmente, cabe agregar que en instancias como las sesiones del Consejo de Estado, la Comisión Ortúzar y la Comisión Conjunta, el discurso neoliberal radical que terminó por imponerse no siempre fue aceptado unánimemente por sus integrantes, incluso, existían en algunos miembros aprensiones al hecho de desligar al Estado de su rol regulador y coordinador en educación, lo que devela que el paso ulterior de un discurso a otro no estuvo exento de vicisitudes que el grupo economicista neoliberal tuvo que soslayar. 


\section{REFERENCIAS}

ACADEMIA SUPERIOR DE SEGURIDAD NACIONAL. Seguridad Nacional. Santiago: Editorial Universitaria, n. 3, 1976.

ACADEMIA SUPERIOR DE SEGURIDAD NACIONAL. Seguridad Nacional. Santiago: Editorial Universitaria, n. 5, 1977.

CHILE. Presidente, Mensaje presidencial 11 de septiembre 1974 - 11 de septiembre 1975: S. E. el presidente de la república General de Ejército Augusto Pinochet Ugarte informa al país. Santiago: Talleres Gráficos de Gendarmería de Chile, 1975.

CHILE. Un año de reconstrucción. 11 de septiembre 1973 - 11 de septiembre 1974: el Jefe Supremo de la nación General de Ejército Augusto Pinochet Ugarte informa al país. Santiago, 1974.

COMISIÓN DE ESTUDIOS DE LA NUEVA CONSTITUCIÓN POLÍTICA DE LA REPÚBLICA DE CHILE. Actas... Santiago, 1975.

CONSEJO DE ESTADO. Actas oficiales 1978-1979. Santiago, 1978-1979.

EL MERCURIO. Directiva para la educación se imparte por orden de la Junta. Santiago, 13 de enero de 1974.

JUNTA DE GOBIERNO. Declaración de principios del Gobierno de Chile. Santiago: La Junta, 1974.

. Objetivo Nacional de Chile. Santiago: Impresora Filadelfia, 1975.

MINISTERIO DE EDUCACIÓN PÚBLICA. Documento $N^{\circ}$ 4: Descripción de la organización del trabajo de evaluación a nivel central. Santiago: Superintendencia, 1974.

MINISTERIO DE EDUCACIÓN PÚBLICA. Directiva n. 87. Santiago: [s.n.], 1973.

MINISTERIO DEL INTERIOR. Constitución Política de la República de Chile. Santiago: Diario Oficial de la República de Chile, 1980.

MINISTERIO DEL INTERIOR. Constitución Política de la República de Chile. Santiago: Biblioteca del Congreso Nacional, 1925.

OFICINA DE RELACIONES INTERNACIONALES. La Educación en Chile, 1979-1980. Informe de Chile a la $38^{\circ}$ reunión de la Conferencia Internacional de Educación, Ginebra, 10-19 noviembre 1981. Santiago: Ministerio de Educación Pública, Oficina de Relaciones Internacionales, 1981.

PÉREZ NAVARRO, Camila. La escuela bajo el Régimen Militar: la experiencia educativa en contexto autoritario. En: COX, Cristián (Ed.). Historia de la Educación en Chile (1810 - 2010). Santiago, Chile. Tomo IV. (en prensa).

PROGRAMA INTERDISCIPLINARIO DE INVESTIGACIONES EN EDUCACIÓN (PIIE). Las transformaciones educacionales en el Régimen Militar. Santiago: PIIE, 1984. 
Estado Docente, subsidiariedad y libertad de enseñanza. El proceso de privatización educacional en Chile desde la perspectiva de los actores (1973-1990)

QUER, Santiago A. Análisis crítico de la Directiva para la Educación. Santiago: Escuela de Educación Universidad Católica de Chile, 1974.

SESIONES CONJUNTAS DE LAS COMISIONES LEGISLATIVAS. Actas oficiales 1987-1989. Santiago, 1987-1989.

SUPERINTENDENCIA DE EDUCACIÓN. Plan operativo nacional y regional 1975, sector Educación. Santiago: Ministerio de Educación Pública, La Superintendencia, 1975.

\section{Sobre os autores:}

Camila Pérez-Navarro: Candidata a Doctora en Educación, Facultad de Educación, Pontificia Universidad Católica de Chile. E-mail: camila.perez@uc.cl

Andrés Rojas-Murphy: Sociólogo, Universidad Alberto Hurtado. E-mail: andresrojasmt@gmail.com

\section{Recebido em maio de 2017.}

\section{Aprovado para publicação em junho de 2017.}


